\title{
Justicia Social e Inequidad en la Formación Científica y Tecnológica de Jóvenes Rurales en la Región Maya de México: El Caso de Mex
}

\author{
Social Justice and Inequity in the Scientific and Technological \\ Training of Rural Youth in the Mayan Region of Mexico: The \\ Case of Mex
}

\author{
Roger J. González González * \\ Edith J. Cisneros-Cohernour
}

Universidad Autónoma de Yucatán, México

\begin{abstract}
El propósito de este artículo es examinar los retos y las experiencias de Mex, un joven proveniente de una comunidad rural que se forma en ciencia y tecnología en la región maya de México, así como los significados que tiene dejar su comunidad para continuar con su formación académica. Para ilustrar estos aspectos se desarrolla un estudio de caso cualitativo, permitiendo identificar cuestiones críticas en la formación científica y tecnológica que pueden reproducirse en poblaciones más amplias de jóvenes de comunidades rurales en la región maya de México, quienes en la mayoría de los casos tienen que migrar de sus comunidades de origen para ingresar a la educación superior. Los resultados indican que el caso enfrenta retos y problemas vinculados a la baja calidad de la educación en las comunidades rurales en la región maya de México, la formación en ciencia y tecnología en estas regiones, la pobreza, colonialidad, discriminación, modernidad y bullying. Se recomienda estudiar estas cuestiones con poblaciones más amplias de jóvenes originarios de comunidades rurales que cursan carreras profesionales en ciencia y tecnología.
\end{abstract}

Descriptores: Justicia social; Ciencia; Tecnología; Formación de investigadores; Mayas.

The purpose of this article is to examine the challenges and experiences of Mex, a young man from a rural community that is trained in science and technology in the Maya region of Mexico, as well as the meanings of leaving his community to continue his academic training. In order to illustrate these aspects, a qualitative case study is developed, allowing to identify critical issues in the scientific and technological formation that can be reproduced in broader populations of young people from rural communities in the Maya region of Mexico, who in most cases have to migrate from their communities of origin to have access to higher education. The results indicate that the case faces challenges and problems related to the low quality of education in rural communities in the Mayan region of Mexico, training in science and technology in these regions, poverty, coloniality, discrimination, modernity and bullying. It is recommended to study these issues with broader populations of young people from rural communities pursuing careers in science and technology.

Keywords: Social justice; Science; Technology; Research training; Mayas.

\section{*Contacto: rogr.gonzalez@gmail.com}

ISSN: 2254-3139

www.rinace.net/riejs/ revistas.uam.es/riejs
Recibido: $\quad 3$ de septiembre 2019

$1^{\text {a }}$ Evaluación: 21 de noviembre 2019

$2^{\text {a }}$ Evaluación: 9 de enero 2020

Aceptado: 1 de febrero 2020 


\section{Revisión de la literatura}

Desde una perspectiva moderna de desarrollo, diferentes teóricos afirman que el acceso a la educación y la acumulación de capital humano son los medios más seguros para que cualquier sociedad aspire al progreso económico y social, pues bajo esta postura, se ha encontrado que el nivel educativo de una comunidad es el factor de mayor influencia en el desarrollo económico de los individuos que la integran. Aunado a ello, diferentes investigaciones han constatado que la ciencia y la educación tienen un valor fundamental como factores que contribuyen al desarrollo (Abdi y Guo, 2008, Ayres, 1995; Mochi, 2006; Munir et al., 2017; Muñoz, 2001; Ozturk, 2001).

El formar capital humano especializado en investigación científica y tecnológica es un tema prioritario en la agenda pública de los gobiernos y los organismos internacionales. En este sentido, la formación en investigación se puede definir como aquellas actividades que buscan promover la apropiación y el desarrollo de competencias necesarias para poder realizar de manera óptima actividades propias de la investigación científica, el desarrollo tecnológico y la innovación en sectores académicos y productivos (Guerrero, 2007).

A pesar de los esfuerzos de organizaciones e instituciones tanto públicas como privadas, nacionales e internacionales, una de las principales preocupaciones de muchos países es el alejamiento de los jóvenes de la ciencia, pues este fenómeno es preocupante para cualquier sociedad que intente elevar sus capacidades en ciencia y tecnología, así como su alfabetización científica (Osborne, Simon y Collins, 2010). Por ello, estudios como el de Ríos (2014) permiten afirmar que las principales carencias en el ámbito de la formación de jóvenes investigadores se vinculan al bajo rendimiento académico y la desmotivación de los estudiantes por continuar con su proceso formativo en investigación, lo cual a su vez genera un escaso interés de los jóvenes por cursar carreras profesionales relacionadas con la Ciencia, la Tecnología, la Ingeniería y las Matemáticas, STEM por sus siglas en inglés (Badri et al., 2016).

En América Latina la formación de investigadores se desarrolla principalmente en las universidades y específicamente se lleva a cabo a través de programas de doctorado, aunque existen esfuerzos por desarrollar las vocaciones hacia la ciencia en el nivel de pregrado a través de la creación de estrategias para la formación de investigadores como elemento prioritario de las políticas nacionales (Guerrero, 2007), pues a nivel pregrado "la formación para la investigación permite adquirir nuevas maneras de comprender la realidad para poder enfrentarse a las dificultades de la producción de conocimientos" (Escamilla, 2014, p. 839). Aunado a lo anterior, se ha encontrado que el enfoque predominante en la formación en investigación es anglosajón, modelo que se caracteriza por el énfasis en la formación en matemáticas, química, física y biología (Moreno, 2016), por ello se requiere la revisión de trabajos empíricos en Latinoamérica sobre el fomento de la actividad científica, así como el comparativo con los países altamente industrializados.

La formación de capital humano en competencias para la investigación, en América Latina está estrechamente relacionada a la normativa y las políticas públicas en ciencia y tecnología, así como al financiamiento que garantice la continuidad de los proyectos (Sebastián, 2003); esto impacta de manera directa en el acceso que la población en general, y sobre todo la población de comunidades rurales, puede tener a la educación científica y tecnológica. Muestra de ello es que según datos reportados por la Organización de las 
Naciones Unidas para la Educación, la Ciencia y la Cultura (UNESCO, 2015), en la región de América Latina habitan un total de 288.500 investigadores, lo cual refleja una cifra alarmantemente baja en comparación con los Estados Unidos de América y Canadá (1.433.300 de investigadores entre ambos países); pues como se observa en el cuadro 1, en América Latina apenas se encuentra el 3,6\% del total de investigadores en el mundo, mientras que en regiones como la Unión Europea se encuentra cerca de la cuarta parte de la población total de investigadores en el planeta.

Cuadro 1. Población de investigadores en la Unión Europea, América del Norte y América Latina

\begin{tabular}{lc}
\hline \multicolumn{1}{c}{ REGIÓN } & INVESTIGADORES (N) EN EL MUNDO \\
\hline Unión Europea & $1.726 .300(22 \%)$ \\
América del norte & $1.433 .300(18.5 \%)$ \\
América Latina & $288.500(3.6 \%)$ \\
\hline Fuente: Modificado de UNESCO (2015), Informe de la UNESCO sobre ciencia, hacia el 2030.
\end{tabular}

Fuente: Modificado de UNESCO (2015), Informe de la UNESCO sobre ciencia, hacia el 2030.

Con relación a la situación de México, el tema de la formación de investigadores científicos y tecnólogos presenta notables carencias, algunos de los principales factores que impactan en este ámbito son: a) los modelos de formación de investigadores occidentales que han predominado en el país a través de los años, b) la baja inversión pública y privada, c) la evaluación descontextualizada en todos los niveles y actores del sistema educativo nacional, basada en pruebas estandarizadas e indicadores internacionales, y d) la inconsistencia entre la realidad de la población y los requerimientos de la ciencia y tecnología “formal” (Acuña y Mérida, 2015; González González, 2019; Rivas, 2004).

Dichas carencias se ven reflejadas en los indicadores y en las distintas regiones que componen a México, pues en un análisis realizado con datos del Sistema Nacional de Investigadores (SNI) del Consejo Nacional de Ciencia y Tecnología (CONACYT, 2018), se aprecia que el centro del país es la zona en la que se concentra el grueso de investigadores con 19, 919; en segundo lugar se encuentra la región norte del país, en la que el desarrollo industrial y tecnológico contribuyen al desarrollo de capacidades en investigación científica, tecnológica y de innovación, pues en dicha región se cuenta con 5,068 investigadores registrados en el SNI.

Estos datos permiten afirmar que incluso internamente el número de investigadores y en consecuencia el desarrollo científico en México no son equitativos, pues como se observa en la figura 1 el sur del país se caracteriza por el escaso número de investigadores, ya que en estados como Yucatán, Quintana Roo, Campeche, Tabasco, Guerrero, Oaxaca y Chiapas apenas se encuentra a 2.001 investigadores registrados en el Sistema Nacional de Investigadores, cifra distante al centro y norte de México. Es importante tener en cuenta que estados como Yucatán, Quintana Roo, Campeche, Tabasco y Chiapas conforman la zona maya de México, una región caracterizada por los altos niveles de pobreza y marginación de su población (Bracamonte y Lizama, 2003; Robles-Zavala, 2010). 


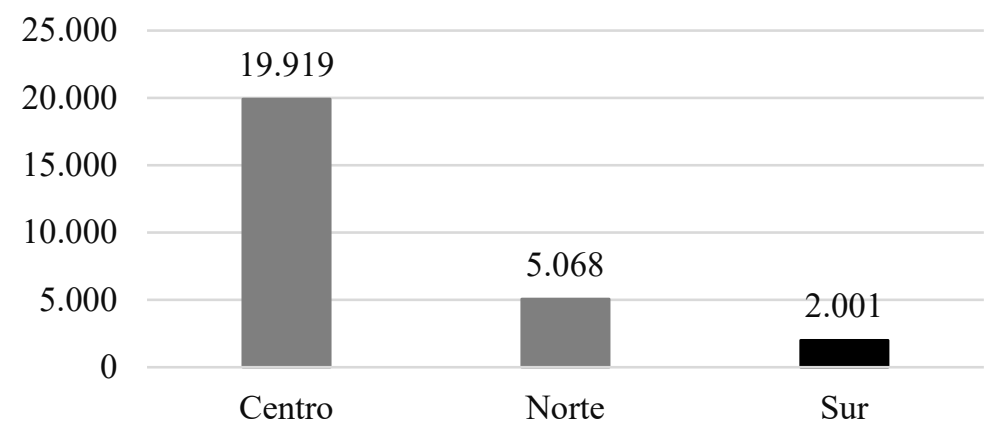

Figura 1. Número de investigadores SNI por región en México Fuente: Elaboración propia.

Todos estos indicadores evidencian que si bien América Latina y en particular México son regiones en las que el desarrollo científico, tecnológico, la innovación y la formación de capital humano son carentes, el fenómeno se agrava a nivel regional y aún más para el caso del estado de Yucatán, pues se cuenta con apenas 659 investigadores registrados en el Sistema Nacional de Investigadores (figura 2), lo cual representa el 2,3\% de los 28,634 especialistas en investigación científica y tecnológica registrados en todo México (CONACYT, 2018). Es necesario señalar que en la base de datos 2018 del Sistema Nacional de Investigadores, de la cual la cual se toman las cifras antes mencionadas, se encontraron 1,645 investigadores que no se ubican en algún estado en particular.

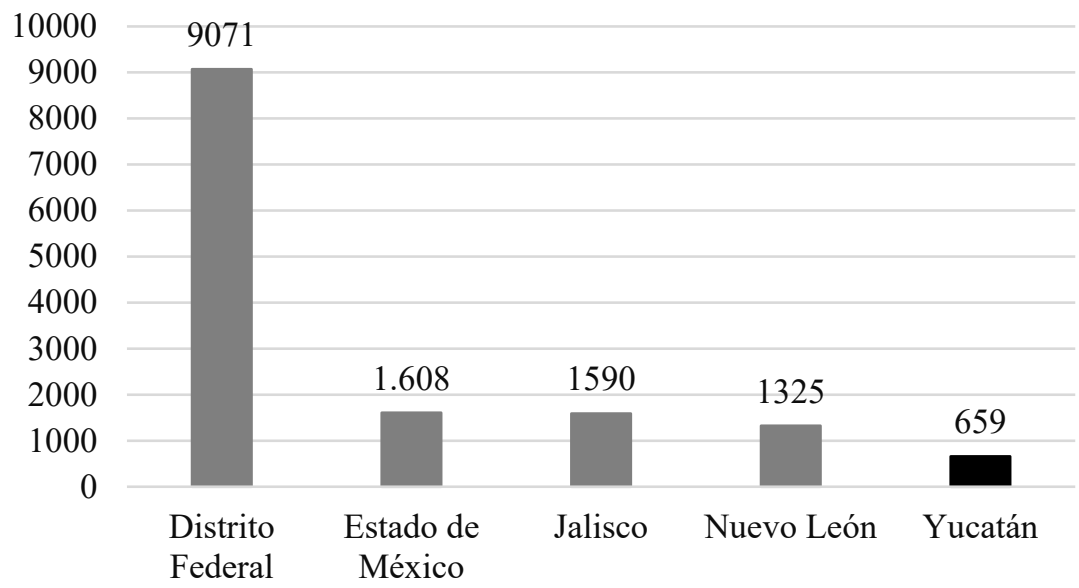

Figura 2. Número de investigadores SNI por estado Fuente: Elaboración propia.

Por otro lado, diferentes organismos internacionales han pretendido cuantificar y comparar el desempeño en ciencia de los estudiantes de la región maya de México a través de instrumentos y pruebas estandarizadas como la prueba PISA (Programme for International Student Assessment), una prueba que mide los conocimientos en lectura, matemáticas y ciencias de estudiantes de 15 años que cursan la educación secundaria o media superior en países pertenecientes a la OCDE (Organización para la Cooperación y el Desarrollo Económicos), cuya última versión se centró en el área de ciencias (Instituto Nacional para la Evaluación de la Educación, 2017); sin embargo, estas herramientas únicamente evidencian las desigualdades que predominan en la educación que se imparte 
en América Latina y cómo se evalúa a los estudiantes, pues este instrumento no es consistente con las características y el contexto sociocultural de los jóvenes de la región maya y rural de México, perpetuando así la inequidad educativa y por ende en la formación en ciencia y tecnología que persiste en Latinoamérica y en México (Donosio et al., 2013; Tiramonti, 2014).

Es importante abordar estas problemáticas, pues se ha encontrado que el acceso a la educación y en especial a la formación en ciencia y tecnología, son algunos de los principales problemas en las zonas rurales (Atchoarena y Gasperini, 2004), siendo la inequidad, la baja calidad de la educación superior, científica y tecnológica problemas que caracterizan al sistema educativo en México (Acuña y Pons, 2016; De Ibarrola, 2012; Fernández, 2017). Estas características contribuyen de manera significativa a la desigualdad social y obstaculizan el desarrollo de las comunidades rurales, por ello es necesario generar estrategias que promuevan la integración y la igualdad de los jóvenes de comunidades rurales con relación a la educación formal, científica y tecnológica, ya que en la actualidad en México existen enormes injusticias respecto a la educación en el ámbito rural (Schmelkes, 2013).

\section{Método}

Debido a lo anterior, el propósito de esta investigación fue examinar los retos y las experiencias de un joven del sur de Yucatán que se forma en ciencia y tecnología, así como los significados que tiene dejar su comunidad rural para continuar con su formación académica, desde la perspectiva de los participantes.

Para ello se llevó a cabo un estudio de caso cualitativo, pues a través de él se busca la comprensión de un caso particular y de su contexto para poder entender su desarrollo en ciertas circunstancias y actividades (Stake, 1995). Al respecto, se considera pertinente este diseño de investigación pues los estudios de caso permiten analizar la conducta de las personas involucradas en el tema de investigación en su propio contexto (Simons, 2011; Yin, 1994).

En cuanto al tipo de estudio de caso, la presente investigación se desarrolló en la modalidad de caso instrumental, ya que en ocasiones este tipo de caso ayuda en la comprensión general de un fenómeno a través del estudio de un caso particular (Stake, 1995).

\section{Diseño del caso}

El estudio se enfocó en Mex, un joven de 18 años originario de una comunidad rural del sur de Yucatán, ubicada en la zona maya de México. Vale la pena señalar que el joven se autodenominó Mex debido a su interés y participación en actividades de investigación científica de la especie marina Limulus polyphemus, mejor conocida en Yucatán como Cacerolita de mar, cuyo nombre maya es Mex. En la actualidad, el joven cursa la Licenciatura en Biología en la Universidad Autónoma de Yucatán (UADY).

Desde pequeño Mex ha tenido curiosidad por el mundo que lo rodea y los seres vivos, debido a ello desde los 11 años participa en actividades de fomento a la investigación como el programa Jóvenes Talentos (Yucatán), concursos de ciencia, ferias de ciencia, olimpiadas del conocimiento, entre otras actividades que lo han motivado a formase en el ámbito de la investigación científica y tecnológica. 
Es necesario señalar que el programa Jóvenes Talentos es una iniciativa del gobierno federal de México, a través de la cual se "busca promover, difundir, fomentar y estimular en niños y jóvenes talentosos de todos los niveles académicos, vocaciones por la actividad científica y tecnológica, a través de propuestas institucionales" (CONACYT, 2017, p. 11). Mex ha participado en este programa a lo largo de nueve años, durante la educación secundaria, media superior e incluso durante sus estudios de licenciatura, generando una marcada influencia en su desarrollo académico y en investigación.

El joven realizó toda la educación básica y media superior en su comunidad de origen, mudándose a la ciudad de Mérida (capital del estado de Yucatán) para cursar la educación superior en el Campus de Ciencias Biológicas y Agropecuarias de la UADY.

Con el fin de ilustrar las particularidades, contextos y antecedentes del caso, en la Figura 3 se observa el diseño del caso de Mex, con base en la propuesta de Stake (2006) para el diseño de casos cualitativos. Como se aprecia, en la investigación se tomaron en cuenta los múltiples contextos en los que interactúa el joven, su trayectoria académica, su participación en actividades de fomento a la investigación científica y la información recabada a través de la interacción con sujetos clave en la vida personal, comunitaria, académica y de fomento a la investigación de Mex.

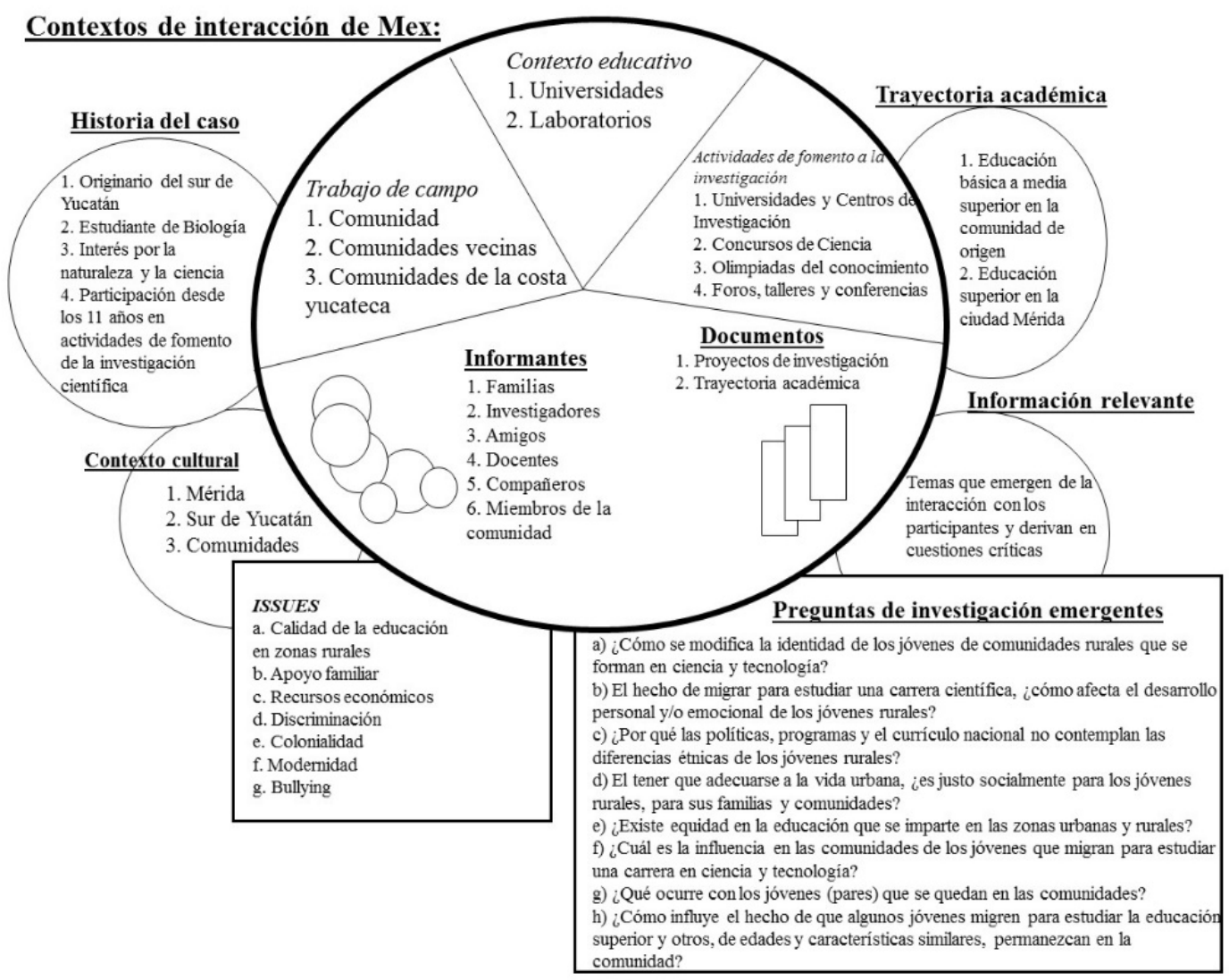

Figura 3. Diseño del caso de Mex

Fuente: Realizado a partir de Stake (2006, p. 5).

A través de los contextos, antecedentes e interacción con los participantes en el estudio, como resultado de la investigación emergieron cuestiones críticas como: 1) Calidad de la educación en comunidades rurales, 2) Apoyo familiar, 3) Recursos económicos, 4) 
Discriminación 5) Colonialidad, 6) Modernidad y 7) Bullying. En la sección de análisis de resultados se abordará con mayor precisión estas cuestiones y cómo fueron emergiendo en el estudio de caso.

\section{Participantes}

En la investigación cualitativa, que se basa en el análisis de experiencias con relación a un fenómeno, es necesario que los participantes interpreten lo que está ocurriendo, pues es importante que la interpretación provenga de las personas, el "origen de datos" (Stake, 2010). Por ello los participantes en esta investigación fueron Mex, sus padres, un amigo del joven que de igual forma participa desde los 11 años en actividades de fomento a la investigación, amigos de su comunidad, compañeros de la licenciatura, docentes e investigadores con los que se ha formado en el desarrollo de proyectos de investigación científica y tecnológica.

\section{Técnicas para la colecta de datos}

La recolección de datos se llevó a cabo durante trece meses, iniciando en diciembre de 2017 y concluyendo en enero de 2019. Para ello, se recurrió a diversas técnicas que a continuación se describen:

Análisis documental: Para la mejor comprensión del caso y sus contextos, se recurrió al análisis de documentos como informes académicos del joven, proyectos de investigación elaborados por Mex, informes sociodemográficos y estadísticos de la comunidad de procedencia del participante, así como el plan de estudios de la licenciatura que actualmente cursa.

Entrevistas grupales: Se realizaron entrevistas grupales con tres grupos de participantes en el estudio, los cuales se describen a continuación:

- Familia. Durante los trece meses de la coleta de datos, se realizaron cinco entrevistas grupales en las cuales participaron los padres del joven.

- Amigos de la comunidad. Tuvieron lugar tres entrevistas grupales con seis amigos de la comunidad, quienes proveyeron información valiosa acerca del contexto y los antecedentes de Mex en el ámbito académico, de la ciencia y la tecnología.

- Otros jóvenes investigadores. Se llevaron a cabo dos entrevistas grupales con tres amigos de Mex, quienes se forman en el ámbito de la investigación científica a través de programas formales y actividades de fomento a la investigación como concursos, ferias, congresos y foros académicos.

Entrevistas a profundidad: Las entrevistas a profundidades fueron la técnica básica para poder identificar y corroborar las cuestiones críticas que emergieron del caso. Debido a ello, se llevaron a cabo un total de ocho entrevistas a profundidad con Mex, en las que se fueron abordando desde temas básicos referentes a la vida académica y el contexto del joven, hasta temas de mayor complejidad que fueron emergiendo durante la colecta de datos. No se consideran entrevista formales, pero las pláticas cotidianas y la convivencia con el joven contribuyeron a la comprensión del caso y las cuestiones críticas que fueron emergiendo.

Entrevistas semiestructuradas: Se llevaron a cabo tres entrevistas semiestructuradas con tres profesores-investigadores de instituciones como la Universidad Autónoma de 
Yucatán y la Universidad Nacional Autónoma de México, quienes han trabajado como asesores de Mex en el desarrollo de proyectos de investigación. Adicionalmente se realizó una entrevista semiestructurada con un amigo de Mex, quien al igual que él, desde los 11 años participa en actividades de fomento a la investigación.

Observaciones: Durante los trece meses de la recolección de datos, se realizaron observaciones en los diversos contextos de interacción del caso, tales como la comunidad, la escuela y las actividades de fomento a la investigación en las que Mex participaba. La observación en diversos contextos y momentos de la vida del joven fue básica para comprender las múltiples realidades que constituyen el caso.

Como se observa en el cuadro 2 se realizaron 22 entrevistas en las que participaron 16 personas, quienes proporcionaron información acerca de los antecedentes del caso y sus contextos. A pesar de que se utilizaron diversas técnicas, la observación y las entrevistas fueron imprescindibles, pues se consideran el medio principal para conocer las múltiples realidades en los estudios de caso (Stake, 1995).

Cuadro 2. Número de participantes y entrevistas realizadas como parte del proceso de recolección de datos en el estudio

\begin{tabular}{lcc}
\hline & ENTREVISTAS (N) & PARTICIPANTES (N) \\
\hline Padres de Mex & 5 & 2 \\
Amigos de la comunidad & 3 & 6 \\
Otros jóvenes investigadores & 2 & 3 \\
Mex & 8 & 1 \\
Profesores investigadores & 3 & 3 \\
Amigo de Mex & 1 & 1 \\
\hline Total & 22 & 16 \\
\hline
\end{tabular}

Fuente: Elaboración propia.

Para las entrevistas se diseñaron preguntas generales, de las cuales surgieron otras para profundizar en la información proporcionada por los participantes. Las preguntas generales realizadas a los participantes se basaron en temas como experiencias durante la formación en ciencia y tecnología, retos durante la formación en ciencia y tecnología e influencia del lugar de procedencia de los jóvenes que se forman en ciencia y tecnología.

Vale la pena señalar que las conversaciones en la plaza principal y en algunos sectores de la comunidad, conocidas informalmente como vagabundeo (Rodríguez, Gil y García, 1996) consistieron en un acercamiento de carácter informal, que proveyeron información del escenario, opiniones y características de la zona en la que se desarrolla el problema.

\section{Triangulación del estudio}

La investigación cualitativa es por sí misma un enfoque multimétodo, pues la utilización de múltiples métodos o técnicas asegura la comprensión profunda del fenómeno a través de su triangulación. Por ello la triangulación no es una herramienta o técnica de validación, sino una alternativa a la validación (Flick, 2002).

En esta investigación el proceso de triangulación se realizó a través de fuentes de información, pues en consistencia con Stake (1998, p. 98) en la triangulación por fuentes de información se observa "si el fenómeno o caso sigue siendo el mismo en otros momentos, en otros espacios o cuando las personas interactúan de forma diferente”. 


\section{Resultados}

El estudio de caso de Mex permite entender muchas de las cuestiones complejas que ha vivido como parte de su formación educativa, en ciencia y tecnología, así como identificar cuestiones críticas que en ocasiones pueden llegar a enfrentar los jóvenes de comunidades rurales que se forman en ciencia y tecnología en la región maya de México. Adicionalmente, el estudio permite identificar aspectos que no son tomados en cuenta desde el planteamiento de las políticas públicas en educación, ciencia y tecnología.

Como resultado de la investigación emergieron siete cuestiones críticas del estudio de caso (figura 4), las cuales se vinculan a los significados, experiencias y retos que el joven ha tenido que afrontar durante su formación académica, científica y tecnológica.

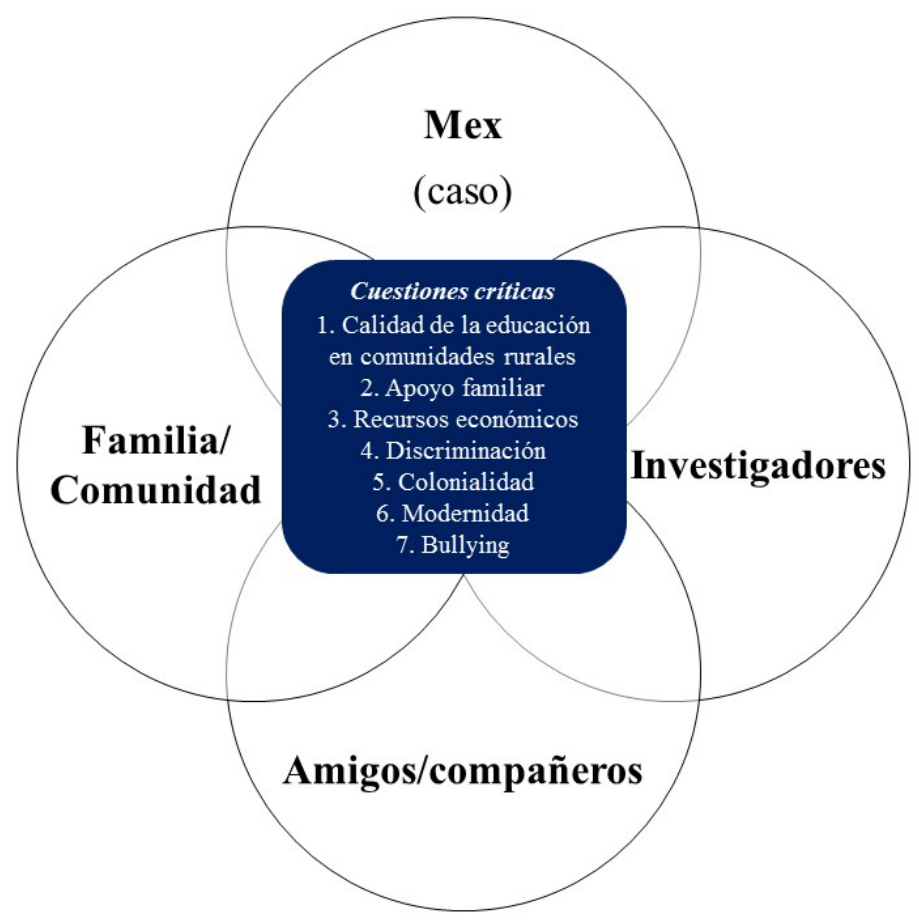

Figura 4. Temas críticos que emergen del estudio de caso de Mex

Fuente: Elaboración propia.

El análisis de la información recabada a través de las observaciones, entrevistas y pláticas informales se realizó por medio de la técnica de diagramas de afinidad, la cual permite agrupar y categorizar datos de manera natural, reuniendo la información a través de temas generales en los cuales se agrupan temas que se relacionan entre sí (Kawakita, 1977). Es importante señalar que históricamente la ciencia occidental ha proporcionado metodologías estructuradas y experimentales para el análisis de datos, sin embargo a diferencia de ellas los diagramas de afinidad son una alternativa para el análisis de información basada en observaciones no estructuradas (Scupin, 1997).

A continuación se presentan los resultados agrupados por temas que emergieron en la investigación, para ello, se categorizaron las cuestiones más relevantes y de mayor coincidencia entre los participantes en el estudio. 


\title{
3.1. La calidad de la educación rural, una mirada a través de la comunidad
}

A través de la interacción, observaciones y entrevistas con los padres de Mex, se encontró que desde su perspectiva y vivencias los temas más importantes que han influido en la formación en ciencia y tecnología del joven son los siguientes:

- Migración

- Calidad de la educación en la comunidad

- Apoyo familiar

- Recursos económicos

- Compañerismo

- Apoyo municipal

- Saberes originarios como aprendizajes científicos

El tema al que los padres dan mayor peso y comentan que ha tenido mayor influencia en la formación de Mex, así como en su trayectoria en la ciencia y tecnología ha sido la calidad de la educación que se imparte en la comunidad, pues su discurso y experiencias se vinculan a la baja calidad de la educación en las comunidades rurales con relación a las zonas urbanas. $\mathrm{Al}$ respecto, los padres comentan:

\begin{abstract}
Hay muchas diferencias... el tipo de educación que llevan los del pueblo es más baja en calidad que la de Mérida... en sí lo que son las materias, es más avanzado lo que les enseñan a los muchachos de Mérida a los de acá, como que el contexto en los libros no es el mismo... en ciencia y tecnología es más avanzado el nivel que tiene la ciudad al del pueblo, no sirven los laboratorios.
\end{abstract}

Los padres hablan de las diferencias en la calidad de la educación que se imparte en zonas rurales y zonas urbanas, sobre todo en el ámbito de la ciencia y tecnología, pues comentan que en las instituciones de educación pública no se cuenta con materiales de laboratorio, infraestructura y tampoco se capacita a los docentes para integrar temas científicos y tecnológicos a su práctica pedagógica.

\subsection{Apoyo familiar en la formación en investigación}

$\mathrm{Al}$ interactuar y entrevistar a tres de los investigadores con los que Mex ha trabajado durante su formación en investigación, se encontró que desde su perspectiva existen temas que son fundamentales y han influido en la formación de Mex en el ámbito científico. Estos temas son:

- Calidad de la educación en la comunidad

- Apoyo familiar

- Recursos económicos

- Comunicación

Los investigadores coincidieron en que un elemento fundamental en la formación científica y tecnológica de Mex ha sido el apoyo de su familia, pues desde la educación básica y su inicio en las actividades vinculadas a la formación de jóvenes investigadores, ha recibido un acompañamiento muy cercano de sus padres y hermanos menores. Al respecto una de las investigadoras con las que Mex incursionó en las ciencias sociales, durante su proceso formativo en bachillerato, comenta: 
Involucra a su familia en sus actividades de formación profesional de la biología... Yo creo que eso también es importante, el apoyo familiar, el interés de la familia por lo que le gusta profesionalmente hacer al hijo, los papás lo apoyan muchísimo, le dan cierta libertad...

... Sí influye mucho que la familia potencie sus capacidades...

\subsection{Otras realidades rurales en la formación para la investigacion: Los amigos de Mex}

Además de las observaciones en la escuela y la convivencia con los compañeros de clase de Mex, como se comentó en la sección de técnicas para la colecta de datos, se tuvo la oportunidad de interactuar y entrevistar a jóvenes de comunidades rurales que han participado en actividades de fomento a la investigación. Los temas que emergieron de esta interacción fueron los siguientes:

- Calidad de la educación en la comunidad

- Apoyo familiar

- Recursos económicos

- Compañerismo

- Migración

- Modernidad

- Colonialidad

- Colonialidad interna

El tema de los recursos económicos que las familias invierten para que los jóvenes puedan participar en actividades de iniciación en la ciencia, es uno de los aspectos más relevantes y de mayor coincidencia entre los participantes, pues como comentan puede llegar a representar una preocupación para ellos. Al respecto un amigo de Mex, proveniente de la misma comunidad indica:

Primero me di cuenta sobre la economía, porque notaba que aquí en la casa con tal de
pagar que yo vaya a una clase de Jóvenes Talentos... me di cuenta de que sí era difícil
para mi familia, luego cuando tuve que continuar con las siguientes experiencias de
por decir viajar hasta Sisal [Sisal es una comunidad en la que realizaron un proyecto
de investigación los jóvenes, ubicada en la costa de Yucatán, a $114 \mathrm{~km}$. de su
comunidad de origen], sí notaba que era un gasto bastante grande y eso sí me
preocupaba.

Es importante señalar que en la comunidad de Sisal se encuentra un Centro de Investigación de la Universidad Nacional Autónoma de México, al cual los jóvenes asistían para trabajar en un proyecto de investigación.

Entre los participantes se encuentra un amigo de Mex llamado Raúl, quien proviene de una comunidad rural ubicada en la parte noroccidental de Yucatán y que al igual que Mex, participa en actividades de fomento a la ciencia y tecnología desde los 11 años. A través de la interacción y entrevistas con Raúl emergieron temas como la Modernidad y Colonialidad, cuestiones críticas que se pudieron confirmar con el propio Mex al final del estudio y que guardan una estrecha relación entre sí, siendo la colonialidad constitutiva de la modernidad (Mignolo, 2000). Referente a estos temas Raúl comenta:

Como que te desenvuelve y te da confianza contigo mismo [vivir en la ciudad]...pero a la vez, como que siempre recordándote dices, oye y mis amigos que se quedaron en 
mi pueblo, pues qué pasó con ellos, qué habría pasado... y esa pregunta siempre me la he hecho...¿Qué habría pasado si yo me hubiese quedado en mi comunidad, si yo nunca hubiese tomado Jóvenes Talentos y la prepa la hubiese hecho allá, cómo hubiese estado?

De hecho igual a Mex le pasó y a varios de mis amigos...te da un poco de nostalgia ver lo que ya dejaste, lo que fue tu pueblo, lo que es una casa con mucho más de una familia... salir a la calle y ver como que todos son familiares.

Mex y Raúl, como muchos jóvenes de comunidades rurales en Yucatán que desean cursar la educación superior han tenido que migrar a la ciudad de Mérida, adaptándose a la vida urbana y todo lo que esta implica. Vinculado a estas experiencias emerge el tema crítico de la Colonialidad interna, fenómeno que consiste en la "aparente relación paradójica de los Estados independientes respecto de sus poblaciones colonizadas", así como la diferenciación colonial ejercida por quienes son los encargados de la construcción nacional (Mignolo, 2000; Quijano, 2000, p. 28). Se pudo identificar este tema en una de las entrevistas con Raúl, quien comentó:

Bueno...pues como todo chico que viene del interior [del estado] al establecerse o al pasar más tiempo en la ciudad, sufrí una pequeña crisis de identidad, se podría decir. Más que personal, social se podría decir... que cuando llegas [a tu comunidad]...entre tus amigos te dicen ha mira, ahí viene el meridano, ahí viene el chico de Mérida ...¿̇oye ya se te olvidaron los triciclos?, ahi puros camiones.

En un principio lo tomas a juego, pero pues con la insistencia te das cuenta que hay algo que ya cambió...

La ciudad te cambia, la ciudad pues ya no es lo mismo, y si bien la ciencia me permitió desenvolverme tanto académicamente como urbanisticamente, porque venir a Mérida e irme a una facultad ya no me daba miedo... andar sólo por acá me dejó de dar miedo.

Este tipo de segregación generada por los miembros de la comunidad hacia quien regresa a ella, se conoce como colonialidad interna y se ve reflejada en la experiencia compartida por Raúl, amigo de Mex.

\subsection{Mex, el joven maya que nada en el neoliberalismo}

Como se ha comentado la interacción con Mex duró más de un año, tiempo en el que se pudo caracterizar al caso como un joven con muchas ganas de aprender, incursionar en la investigación y ser un orgullo para su familia y su comunidad. A través de la convivencia, interacción y entrevistas con Mex, se pudo identificar que desde su perspectiva y sus experiencias, los temas clave que emergen del propio joven son los siguientes:

- Calidad de la educación en la comunidad

- Apoyo familiar

- Pobreza

- Discriminación

- Colonialidad

- Bullying

A través de las observaciones en diferentes contextos, pláticas informales y entrevistas con Mex, emergió el tema de la discriminación. Es necesario señalar que esta cuestión crítica no emergió de manera inmediata, ya que es un tema delicado que se pudo abordar 
después de varios meses de observaciones, entrevistas e interacción con el caso. Con relación a la discriminación a la que se ha visto expuesto, Mex comenta:

Tuve una experiencia con un chavo que sí me discriminó bastante por ser de un pueblo y cuando entré a la universidad me lo volvi a topar y por lo mismo, que yo era COBAX [Colegio de Bachilleres de Tucatán], que yo no sabía lo mismo que él veía en su escuela y que él era mucho mejor que yo...siempre que estábamos platicando o algo hacía bromas sobre de dónde venía y sobre lo que sabia, me trataba como si yo fuera inferior a él...tuve ambas experiencias una en la prepa y otra en la secundaria...

...cuando yo llegué a Mérida no sabía sobre plazas [centros comerciales], no sabía sobre nada y ellos conocían eso cada fin de semana, entonces como ellos hablaban de eso y yo no sabía me trataban como de menos...

...hubo una ocasión en la que estábamos también mencionando de qué prepas veníamos y yo dije yo soy de COBAT, soy de una comunidad rural al sur de rucatán. Me dijeron, ah ¿̇enserio?, ¿̇ómo es que lograste salir de ahí?, ¿qué ahí no aprenden nada? Y fue ya cuando sentí el golpe como personal. .

Mex comparte su experiencia al convivir con jóvenes de otras escuelas de procedencia, comunidades y niveles socioeconómicos al ingresar a la educación superior. Como se aprecia el joven fue objeto de discriminación debido a su comunidad de origen y a la institución en la que estudió el bachillerato.

Con relación al hecho de provenir de una comunidad maya y rural del sur de Yucatán, se manifiesta una de las formas de discriminación más fuertes social y culturalmente, pues consiste en discriminar a "un sector de la población que lleva en el cuerpo las marcas de su origen indígena o mestizo, y cuya presencia en la gran ciudad se torna visible”, ya que el concepto de discriminación se vincula estrechamente a la pobreza y marginación social de los individuos (Margulis y Urresti, 1999, p. 9), elementos que como se ha comentado, caracterizan a la población de la zona maya de México. Los modos de discriminación varían dependiendo del momento y el contexto, por lo que Mex se enfrentó al tema de la discriminación que se gesta en el ámbito universitario, la cual es "producida por la presencia de pensamientos y numerosos actos infundados en una ideología racista, por cuestiones étnicas, de color de piel, lengua, o pertenencia a una minoría nacional" (Lara, 2013, p. 2).

El tema del bullying fue una de las cuestiones críticas más fuertes que emergieron de la investigación, pues Mex vivió situaciones de bullying desde el inicio de sus estudios de licenciatura hasta la actualidad, sin embargo el joven comenta que estas conductas han disminuido su frecuencia e intensidad. Entre las reflexiones de Mex con relación al bullying se destaca la siguiente:

El problema que tuve con este chavo es un problema personal, tuvimos mal entendidos, pero se supone que ya hace como seis meses debimos olvidar todo; sin embargo esta persona me sigue amenazando cada vez que puede, e intenta como definir su territorio, por lo que hace muchas cosas que no me agradan y la verdad ya llegó a un punto en el que me desagrada tanto que ya estoy pensando hacer una carta académica para meterla a control académico por las constantes amenazas y la incomodidad que me hace sentir, y todo esto lo tuve que ver hoy porque ayer me llegó otra amenaza y no quiero seguir con esto.

Considero que podría tratarse de un tipo de Bullying o abuso, porque aparte de sus amenazas incluye rumores sobre mi entre sus amigos, y sus amigos los difunden con sus amigos, con sus conocidos y asi se genera una red de rumores alrededor de mí.

Mex vivió situaciones de violencia escolar que él mismo refiere como bullying, fenómeno que de acuerdo con Berger, Karimpour y Rodkin (2008) no se restringe a contextos o 
niveles socioeconómicos específicos, sino que está presente en todas las escuelas, niveles y ámbitos, influyendo de manera negativa en los estudiantes pues incrementa significativamente el rezago educativo, la deserción escolar y afecta el rendimiento académico (Prieto y Carrillo, 2009; Ruiz-Ramírez, et al., 2016; Ruiz-Ramírez, García-Cué, Ruiz y Ruiz, 2018). Se considera de alta relevancia este tema crítico, pues si bien existe basta literatura acerca del bullying escolar y sus implicaciones, es limitada la información que existe con relación a los jóvenes de comunidades rurales y en específico, con relación a quienes se forman en ciencia y tecnología.

Adicionalmente, emergieron temas críticos vinculados a la calidad de la educación rural en Yucatán, el apoyo familiar y la pobreza, poniendo de manifiesto la triangulación de los datos y la consistencia entre la información recabada. En cuanto al tema de la colonialidad y su influencia en la vida personal y académica de Mex, la siguiente sección permite ilustrar estas experiencias en la vida del joven.

\subsection{Y al final, ¿qué ocurrió con Mex?}

Durante el último mes de la recolección de datos la interacción con Mex fue cada vez más complicada, pues el joven empezó a involucrarse en lo que él llama, la vida de la ciudad.

Para poder subsistir y apoyar a sus padres con los gastos que ha generado el migrar a la ciudad, el joven tomó la decisión de empezar a trabajar como cronometrista en carreras deportivas, por lo que en la actualidad tiene una agenda cada vez más cargada, pues tiene que salir de casa a las 4:00 a.m., ir al trabajo y después cumplir con todas las responsabilidades académicas y del hogar que un chico que vive solo en la ciudad tiene.

Con relación al programa Jóvenes Talentos, a través del cual se forma en ciencia y tecnología, Mex comenta que es muy probable que lo abandone, pues la dinámica de su vida actual no le permite cumplir con todas las actividades escolares, científicas y tecnológicas que la escuela y el programa requieren. Durante la última reunión de trabajo, Mex cerró la entrevista con las siguientes frases:

Yo no me siento identificado con la ciudad, si fuera por mí yo seguiría en mi casa, viendo la neblina, los árboles, sintiéndome otra vez en ese ambiente.

Pero estando aquí en Mérida ["la ciudad"] me siento tan atrapado, tan impotente, tan apresurado que a veces me llega a estresar o a fastidiar.

Por ese motivo me he dado el tiempo de salir y conocer Mérida, intentar enamorarme de Mérida, sentir que es mi hogar, ¡Ya llevo un año aquí o un poquito más y aún tengo ese problema!

Mex busca adaptarse a la vida neoliberal de la ciudad, es el único camino que le queda para subsistir (Anderson, 2003), ya que como afirma Valverde (2014), "debe pagar un precio por vivir en la civilización... resulta ser un agregado del sistema, obligado si desea sobrevivir en sociedad, prescindible si no lo logra por incapacidad" (p. 38).

\section{Discusión y conclusiones}

En esta investigación se examinaron los retos y las experiencias de un joven del sur de Yucatán que se forma en ciencia y tecnología, así como los significados que tiene dejar su comunidad rural para continuar con su formación académica. Para ello se llevó a cabo un diseño de investigación por estudio de caso cualitativo, que se sustenta en el paradigma interpretativo (Gutiérrez, Pozo y Fernández, 2002; Lincoln y Guba, 1985; Stake, 1995), 
pues la investigación interpretativa se basa en gran medida en la interpretación de los investigadores, la interpretación de los participantes e incluso de los lectores de los informes de investigación, centrándose en aquellas experiencias de vida que alteran y dan forma radical a los significados que las personas dan a sí mismas y a sus experiencias (Stake, 2010). Como Denzin (2001) afirma, este tipo de investigación "intenta hacer que los significados que circulan en el mundo de la experiencia vivida sean accesibles para el lector. Se esfuerza por capturar y representar las voces, las emociones y las acciones de los estudiados" (p. 1).

Los resultados fueron consistentes con los de otras investigaciones realizadas en la región maya de México, en las que se ha encontrado que las actividades de fomento científico y los programas orientados a la formación de jóvenes investigadores, como lo es el programa Jóvenes Talentos, son la principal estrategia para fortalecer las competencias en ciencia y tecnología de los jóvenes yucatecos (Castillo, 2006; Cisneros-Cohernour y Patrón, 2006; González González, 2017).

El estudio permite entender, a nivel de caso instrumental (Stake, 1988), cuestiones como la desigualdad, el rezago y en consecuencia la marginación educativa que se vive en las zonas rurales del estado de Yucatán. Esto es consistente con el estudio de Mijangos, Canto y Cisneros-Cohernour (2009), donde se afirma que en Yucatán se afronta el grave problema del rezago educativo de los niños y niñas mayas, lo cual los lleva a verse en la necesidad de decidir entre continuar con una educación subestándar, que los margina y no es coherente con su realidad, o dar solución a sus problemas inmediatos y comunitarios.

Estos aspectos son serios pues evidencian la homogenización del sistema educativo nacional, lo que contribuye a perpetuar la desigualdad y la marginación en la educación que se imparte en las zonas rurales de la zona maya de México. Esta marginación y desigualdad, consecuencia del actual sistema educativo neoliberal, a su vez son producto del contexto social en el que los oprimidos han vivido (Mijangos, 2006), recordando que "sería en verdad una actitud ingenua esperar que las clases dominantes desarrollasen una forma de educación que permitiese a las clases dominadas percibir las injusticias sociales en forma crítica" (Freire, 1984, p. 71), por lo que se requiere de un proceso formativo autónomo y que responda a las necesidades de los jóvenes de comunidades rurales en la zona maya de México.

En el mismo sentido, es urgente generar estrategias que permitan elevar la calidad de la educación en las zonas rurales de Yucatán, así como impulsar actividades que promuevan la formación científica y tecnológica de niños, niñas y jóvenes de estas regiones, quienes generalmente se encuentran en situaciones de rezago y marginación. Esta propuesta es relevante pues se observó que las políticas públicas orientadas al fomento de la ciencia y la tecnología, no toman en cuenta el contexto rural y las necesidades culturales de la población rural en la región maya de México.

Por otro lado, es necesario visibilizar los síntomas de discriminación que permean en la educación formal y no formal de los jóvenes de la región, así como generar estrategias para trabajar temas como la discriminación por etnia, lugar de procedencia y antecedentes académicos de los jóvenes yucatecos en todos los niveles educativos.

Se recomienda estudiar con mayor profundidad y poblaciones más amplias las cuestiones que emergieron de este estudio de caso, con base en las cuales se formularon las siguientes preguntas críticas: 
- ¿Cómo se modifica la identidad de los jóvenes de comunidades rurales que se forman en ciencia y tecnología?

- El hecho de migrar para estudiar una carrera científica, ¿cómo afecta el desarrollo personal y/o emocional de los jóvenes rurales?

- ¿Por qué las políticas, programas y el currículo nacional no contemplan las diferencias étnicas de los jóvenes rurales?

- El tener que adecuarse a la vida urbana, ¿es justo socialmente para los jóvenes rurales, para sus familias y comunidades?

- ¿Existe equidad en la educación que se imparte en las zonas urbanas y rurales?

- ¿Cuál es la influencia en las comunidades de los jóvenes que migran para estudiar una carrera en ciencia y tecnología?

- ¿Qué ocurre con los jóvenes (pares) que se quedan en las comunidades?

- ¿Cómo influye el hecho de que algunos jóvenes migren para estudiar la educación superior y otros, de edades y características similares, permanezcan en la comunidad?

Finalmente se proponen respuestas a estas preguntas críticas, las cuales representan las barreras que el caso ha enfrentado a lo largo de su formación en ciencia y tecnología. Las respuestas se construyen desde el estudio de caso, por lo que no son generalizables pero sí ponen de manifiesto la necesidad de estudiarlas con otros jóvenes de comunidades rurales en la región maya de México:

Para adaptarse al mundo de la ciencia y la tecnología, términos propios del modelo de desarrollo neoliberal actual (Lave, Mirowski y Randalls, 2010; Pellizzoni y Ylönen, 2012), el joven rural debe pasar por un proceso de transformación de sus hábitos, costumbres y de su vida en general, modificando sus contextos, personas que forman parte de sus círculos cercanos e incorporando a su vida elementos propios de la modernidad.

Desde el estudio de caso realizado, se puede afirmar que los jóvenes que migran de sus comunidades para estudiar una carrera en ciencia y tecnología, pueden atravesar algún tipo de crisis de identidad. Esta afirmación se realiza con base en las experiencias de los participantes en este estudio.

Con relación a las políticas públicas y programas que promueven la ciencia y la tecnología, así como el currículo nacional, se puede afirmar que no contemplan las diferencias étnicas de los jóvenes rurales, esto se debe a dos factores principales:

- Los diseños homogéneos que se construyen en las altas esferas de los gobiernos e instituciones.

- El desconocimiento por parte de las autoridades acerca de las características y necesidades particulares de los jóvenes rurales con relación a temas educativos, científicos y tecnológicos.

En los modelos recientes de desarrollo lo rural frena el progreso, por lo que las comunidades y sus miembros han tenido que adaptarse a las nuevas demandas del modelo económico-social hegemónico. Por ello, los resultados de este estudio así como los de otras investigaciones (Salas y Velasco, 2013; Steffen y Tarrío, 2010), permiten afirmar que esta adaptación a la modernidad representada por la vida urbana, es injusta para las 
comunidades rurales y sus habitantes, pues en este proceso lo rural pierde flujos de poder, de cultura, territorio y del sentido de comunidad.

Como se ha comentado en este estudio, las regiones rurales en México y particularmente en Yucatán se caracterizan por el rezago y la marginación educativa que tienen que enfrentar sus habitantes. Por ello, se puede concluir que en Yucatán el sistema educativo y los procesos de enseñanza-aprendizaje no son consistentes con los saberes, necesidades e intereses de las poblaciones rurales, por lo que no existe equidad en la educación que se imparte en dichas regiones.

Es complejo determinar la influencia en las comunidades de los jóvenes que migran para estudiar una carrera en ciencia y tecnología, por ello el caso de Mex constituye la primera etapa de una investigación más amplia, en la que como segunda etapa de la investigación se pretende abordar esta cuestión con poblaciones más amplias de jóvenes rurales y sus comunidades de origen. Sin embargo, a través del caso se pudo constatar que la migración de los jóvenes tiene gran incidencia en las familias, amigos y los contextos en los que el joven rural interactuaba antes de dejar la comunidad.

En este estudio se encontró que se generan actitudes de recelo y la construcción de un sentido de colonialidad interna por parte de los jóvenes que permanecen las comunidades, esto se pone de manifiesto cuando los amigos que migraron regresan a la comunidad.

El hecho de que algunos jóvenes migren para estudiar la educación superior y otros, de edades y características similares, permanezcan en la comunidad genera desigualdad en las comunidades rurales, pues independientemente de las capacidades, intereses y actitudes, todos los jóvenes en la comunidad deberían tener la oportunidad de acceder a una educación de calidad y que responda a su contexto. Esta desigualdad y su influencia en los jóvenes de la comunidad serán abordadas en estudios posteriores.

\section{Referencias}

Abdi, A. A. y Guo, S. (2008). Education and social development: An introduction. En A. A. Abdi, y S. Guo (Eds.), Education and social development: Global issues and analyses (pp. 3-12). Sense publishers. https://doi.org/10.1163/9789087904401_002

Acuña, L. A. y Mérida, Y. (2015). Instituto nacional de evaluación de la educación. Una mirada crítica a su nueva función. Revista Mexicana de Investigación Educativa, 20(66), 985-991.

Acuña, L. A. y Pons, L. (2016). Calidad educativa en México. De las disposiciones internacionales a los remiendos del proyecto nacional. Revista Internacional de Investigación en Ciencias Sociales, 12(29), 155-174. https://doi.org/10.18004/riics.2016.diciembre.155-174

Anderson, P. (2003). Neoliberalismo: Un balance provisorio. En E. Sander y P. Gentili (Eds.), La trama del neoliberalismo: Mercado, crisis y exclusión social. (pp. 11-18). Consejo Latinoamericano de Ciencias Sociales.

Atchoarena, D. y Gasperini, L. (2004). Educación para el desarrollo rural: Hacia nuevas respuestas de política. Organización de las Naciones Unidas para la Agricultura y la Alimentación.

Ayres, C. (1995). Economic development: An institutionalist perspective. En J. Dietz (Ed.), Latin America's economic development (pp. 89-97). Lynne Rienner Publishers.

Badri, M., Alnuaimi, A., Mohaidat, J., Al Rashedi, A., Yang, G. y Al Mazroui, K. (2016). My science class and expected career choices. A structural equation model of determinants involving 
Abu Dhabi high school students. International Journal of STEM Education, 3(12), 1-21. https://doi.org/10.1186/s40594-016-0045-0

Berger, C., Karimpour, R. y Rodkin, P. (2008). Bullies and victims at school: Perspectives and strategies for primary prevention. En T. W. Miller (Ed.), School violence and primary prevention (pp. 295-322). Springer. https://doi.org/10.1007/978-0-387-77119-9_15

Bracamonte, P. y Lizama, J. (2003). Marginalidad indígena: Una perspectiva histórica de Yucatán. Desacatos. Revista de Ciencias Sociales, 13, 8-98.

Castillo, C. L. (2006). Evaluación del verano de la investigación científica del PRIORI y de la AMC de la Universidad Autónoma de Tucatán (Tesis de maestría). Universidad Autónoma de Yucatán, México.

Cisneros-Cohernour, E. y Patrón, R. (2014). Preparing young researchers and evaluators through a Mexican summer apprentice programme. US-China Education Review, 4(9), 656-663.

CONACYT. (2017). Informe de actividades de CONACYT enero-septiembre de 2017. http://www.siicyt.gob.mx/index.php/transparencia/informes-conacyt/informe-deactividades/3903-inf-actividades-2017-ene-sep/file.

CONACYT. (2018). Archivo del sistema nacional de investigadores. Investigadores vigentes 2018. https://www.conacyt.gob.mx/index.php/el-conacyt/sistema-nacional-deinvestigadores/archivo-historico.

De Ibarrola, M. (2012). Los grandes problemas del sistema educativo mexicano. Perfiles Educativos, $34,16-28$.

Denzin, N. (2001). Interpretive interactionism. Sage. https://doi.org/10.4135/9781412984591

Donosio, S., Arias, O., Gajardo, C. y Frites, C. (2013). Inequidades invisibles en la educación chilena: Brechas entre estudiantes urbanos y rurales en la prueba pisa de lectura. Educação y Sociedade, 34(125), 1203-1227. https://doi.org/10.1590/S0101-73302013000400010

Escamilla, Z. (2014). Incorporación del pregrado a la investigación en enfermería en México. Revista Cuidarte, 5(2), 837-841. https://doi.org/10.15649/cuidarte.v5i2.125

Fernández, E. (2017). Una mirada a los desafíos de la educación superior en México. Innovación Educativa, 17(74), 183-207.

Flick, U. (2002). An introduction to qualitative research. Sage.

Freire, P. (1984). La importancia de leer y el proceso de liberación. Siglo XXI editores.

Guerrero, M. E. (2007). Formación para la investigación en el contexto universitario. Editorial Universidad Católica de Colombia.

González González, R. J. (2017). Evaluación de un programa de formación de investigadores de nivel medio superior en Yucatán (Tesis de maestría). Universidad Autónoma de Yucatán, México.

González González, R. J. (2019). La formación de jóvenes investigadores en Alemania y México, un estudio comparativo. Revista Educación y Ciencia, 8(51), 41-47.

Gutiérrez, J., Pozo, T. y Fernández, A. (2002). Los estudios de caso en la lógica de la investigación interpretativa. Arbor: Ciencia, Pensamiento y Cultura, 171(675), 533-557. https://doi.org/10.3989/arbor.2002.i675.1045

Instituto Nacional para la Evaluación de la Educación. (2017). México en PISA 2015. Ciudad de México: INEE.

Kawakita, J. (1977). Ido daigaku or free campus university. Research in Higher Education-Daigaku Ronshu, 5, 91-102. 
Lara, G. N. (2013). Discriminación racial dentro del ámbito universitario: Un enfoque sobre la discriminación sutil y manifiesta. Reidocrea, 2, 111-116.

Lave, R., Mirowski, P. y Randalls, S. (2010). Introduction: STS and neoliberal science. Social Studies of Science, 4O(5), 659-675. https://doi.org/10.1177/0306312710378549

Lincoln, Y. S. y Guba, E. G. (1985). Naturalistic inquiry. Sage. https://doi.org/10.1016/01471767(85)90062-8

Margulis, M. y Urresti, M. (1999). La segregación negada: Cultura y discriminación social. Editorial Biblos.

Mignolo, W. (2000). La colonialidad a lo largo y a lo ancho: El hemisferio occidental en el horizonte colonial de la modernidad. En E. Lander (Ed.), La colonialidad del saber: Eurocentrismo y ciencias sociales perspectivas latinoamericanas (pp. 55-86). Consejo Latinoamericano de Ciencias Sociales.

Mijangos, J. C. (2006). Educación popular y desarrollo comunitario sustentable. Una experiencia con los mayas de Yucatán. Ediciones de la Facultad Latinoamericana de Ciencias Sociales.

Mijangos, J. C., Canto, P. J. y Cisneros-Cohernour, E. J. (2009). Introducción. En J. C. Mijangos (Ed.), La lucha contra el rezago educativo. El caso de los mayas en Tucatán (pp. 9-44). Unas Letras Industria Editorial.

Mochi, P. (2006). Globalización, desarrollo local y descentralización. La importancia del conocimiento y la formación de recursos humanos en estos contextos. En C. Girardo, M. Ibarrola, C. Jacinto y P. Mochi (Coords.), Estrategias educativas y formativas para la inserción social y productiva (pp. 145-162). Cinterfor-oit.

Moreno, M. G. (2016). La formación de nuevos investigadores educativos. Revista de la Educación Superior, 45(177), 171-175. https://doi.org/10.1016/j.resu.2016.04.005

Munir, M., Akbar, R. A., Faisal, S., Kayani, A. y Amin, M. (2017). Analysis of socio-economic benefits of education in developing countries: A example of Pakistan. Bulletin of Education and Research, 39(3), 75-92.

Muñoz, C. (2001). Educación y desarrollo económico y social. Políticas públicas en México y América Latina durante las últimas décadas del siglo xx. Perfiles Educativos, 23(91), 7-36.

Osborne, J., Simon, S. y Collins, S. (2003). Attitudes towards science: A review of the literature and its implications. International Journal of Science Education, 25(9), 1049-1079. https://doi.org/10.1080/0950069032000032199

Ozturk, I. (2001). The role of education in economic development: A theoretical perspective. Journal of Rural Development and Administration, 33(1), 39-47.

Pellizzoni, L. y Ylönen, M. (2012). Neoliberalism and technoscience: Critical assessments. Ashgate Publishing.

Prieto, M. y Carrillo, J. (2009). Fracaso escolar y su vínculo con el maltrato entre alumnos: El aula como escenario de la vida afectiva. Revista Iberoamericana de Educación, 49(5), 1-8.

Quijano, A. (2000). Colonialidad del poder, eurocentrismo y América Latina. En E. Lander (Ed.), La colonialidad del saber: Eurocentrismo y ciencias sociales. Perspectivas Latinoamericanas (pp. 246-276). Consejo Latinoamericano de Ciencias Sociales.

Robles-Zavala, E. (2010). Los múltiples rostros de la pobreza en una comunidad maya de la Península de Yucatán. Revista Estudios Sociales, 18(35), 100-133.

Rodríguez, G., Gil, J. y García, E. (1996). Metodología de la investigación cualitativa. Editorial Aljibe. 
Ríos, R. A. (2014). La formación para la investigación en los programas de contaduría: Una función del profesor universitario. Revista Científica General José María Córdova, 12(14), 129-148. https://doi.org/10.21830/19006586.60

Rivas, L. A. (2004). La formación de investigadores en México. Revista Perfiles Latinoamericanos, 12(25), 89-113.

Ruíz-Ramírez, R., García-Cué, J. L., Ruíz, F. y Ruíz, A. (2018). La relación bullying-deserción escolar en bachilleratos rurales. Revista Electrónica de Investigación Educativa, 20(2), 37-45. https://doi.org/10.24320/redie.2018.20.2.1527

Ruíz-Ramírez, R., Zapata-Martelo, E., García-Cué, J. L., Pérez-Olvera, A., Martínez-Corona, B. y Rojo-Martínez, G. (2016). Bullying en una universidad agrícola del estado de México. Revista Ra Ximhai, 12(1), 105-126. https://doi.org/10.35197/rx.12.01.2016.06.rr

Salas, H. y Velasco, P. (2013). Los rostros rurales de dominación en el neoliberalismo actual. Revista Márgenes, 13(10), 7-14.

Schmelkes, S. (2013). Educación para un México intercultural. Revista electrónica Sinéctica, 40, 1-12.

Scupin, R. (1997). The KJ method: A technique for analyzing data derived from japanese ethnology. Human Organization, 56(2), 233-237. https://doi.org/10.17730/humo.56.2.x335923511444655

Sebastián, J. (2003). Estrategias de cooperación universitaria para la formación de investigadores en Iberoamérica. OEI.

Simons, H. (2011). El estudio de caso: Teoría y práctica. Morata.

Stake, R. E. (1988). Case study methods in educational research: Seeking sweet water. En R. M. Jaeger (Ed.), Complementary methods for research in education (pp. 253-278). American Educational Research Association.

Stake, R. (1995). The art of case study research. Sage.

Stake, R. (1998). Investigación con estudio de casos. Morata.

Stake, R. (2006). Multiple case study analysis. The Guilford Press.

Stake, R. (2010). Qualitative research studying how things work. The Guilford Press.

Steffen, M. y Tarrío, M. (2010). Neoliberalismo y crisis agroalimentaria: Adaptación y resistencia de los ejidatarios mexicanos. Revista del Medio Rural Latinoamericano, 56, 11-46.

Tiramonti, G. (2014). Las pruebas PISA en América Latina: Resultados en contexto. Avances en Supervisión Educativa, 20, 1-24.

UNESCO. (2015). Informe de la UNESCO sobre ciencia, hacia el 2030. UNESCO.

Valverde, F. J. (2014). Persona o individuo en el (neo)liberalismo: Algunos fundamentos ideológicos. Revista Pensamiento Actual, 14(22), 29-44.

Yin, R. K. (1994). Case study research: Design and methods. Sage.

\section{Breve CV de los autores}

\section{Roger J. González González}

Licenciado en Educación, Maestro en Investigación Educativa y estudiante del Doctorado Institucional en Ciencias Sociales de la Universidad Autónoma de Yucatán. Es coautor de dos libros enfocados en el desarrollo de competencias digitales por la Secretaría de 
Educación Púbica, así como de diversos capítulos de libro y ponencias en congresos nacionales y en el extranjero. Tiene experiencia docente a nivel licenciatura y posgrado, sus áreas de investigación se centran en la formación de investigadores, la evaluación de programas, la educación intercultural y la tecnología educativa. ORCID ID: https://orcid.org/0000-0003-2876-3539. Email: rogr.gonzalez@gmail.com

\section{Edith J. Cisneros-Cohernour}

Doctora en Evaluación, Administración y Educación Superior por la Universidad de Illinois en Urbana-Champaign. Su investigación se centra en el mejoramiento de la calidad de la educación en las organizaciones educativas, especialmente en las áreas de evaluación, desarrollo profesional y organizacional y los aspectos éticos en evaluación e investigación. Actualmente es Jefa de la Unidad de Posgrado e Investigación en la Facultad de Educación de la Universidad Autónoma de Yucatán y pertenece al nivel dos del Sistema Nacional de Investigadores del CONACYT. ORCID ID: https://orcid.org/O000-0003-2319-1519. Email: ecohernour@gmail.com 